\title{
The relationship between patient engagement and health-related quality of life in patients: a cross-sectional study in general outpatient clinic in Hong Kong SAR, China
}

This article was published in the following Dove Press journal:

Patient Preference and Adherence

\author{
Richard Huan Xu \\ Annie Wai Ling Cheung \\ Eliza Lai Yi Wong \\ Jockey Club School of Public Health and \\ Primary Care, The Chinese University of \\ Hong Kong, Hong Kong, People's \\ Republic of China
}

\begin{abstract}
Introduction: Patient engagement (PE) helps healthcare professionals to collaborate with patients to work together to improve health outcomes. However, the studies of PE and its relationship with health-related quality of life (HRQoL) is very rare in Hong Kong (HK) and China. The aim of this study was to assess the PE level and its association with HRQoL in the general outpatient clinic (GOPC) in Hong Kong.
\end{abstract}

Methods: A cross-sectional survey was conducted in a GOPC in 2017. Patient engagement index (PEI) was used as a valid and reliable instrument to assess the PE level in the primary care setting. EQ-5D-5L HK Chinese version was used to evaluate the patients' HRQoL. ANOVA, ANCOVA and robust linear regression were used to analyse the data.

Results: 686 patients successfully completed the survey (response rate $=64 \%$ ). The mean utility of EQ-5D $(0 \sim 1.0)$ was 0.92 . Male, highly educated and younger respondents got higher utility. For PEI $(0 \sim 100)$, the mean score was 37.02, whereas female (38.03), highly educated and younger respondents performed better. The regression model indicated that there is a positive relationship between PE and HRQoL (beta $=2.66, \mathrm{SE}=5.11, p<0.05$ ). However, after adjusting a series of socio-economic characteristics, the relationship was weakened (beta $=1.77, \mathrm{SE}=4.64, p<0.05$ ) and even insignificant.

Conclusion: This is the first study in HK to assess the PE level and its relationship with HRQoL in the primary care setting. The result indicated that improve PE could bring a positive influence on the HRQoL. However, the PE-HRQoL relationship might be deeply influenced by an individual's physical, psychological or social characteristics.

Keywords: patient engagement, health-related quality of life, general outpatient clinic, Hong Kong, China

\section{Introduction}

In the last decades, the world's population has increased, aged and become increasingly afflicted with diseases (chronic disease), thus posing a great threat to our healthcare system. ${ }^{1}$ Given the potential scope, intensity and duration of these challenges, we believe that the current healthcare pattern cannot address these problems. The doctor-centred care pattern has been questioned; although it is transforming slowly, new patient-centred care is slow to develop. ${ }^{2,3}$ The difficulty of providing services based on patients' preferences and needs is one of the key sources of substantial frustration for professionals, administrators and policymakers in providing effective healthcare services. ${ }^{4,5}$
Correspondence: Eliza Lai Yi Wong Jockey Club School of Public Health and Primary Care, The Chinese University of Hong Kong, Hong Kong, People's Republic of China

Tel +852 22528772

Email lywong@cuhk.edu.hk 
Currently, unlike some synonymous concepts, such as activation and involvement, an increasing number of studies have recognised that patient engagement (PE), as an umbrella and culturally sensitive concept, is a crucial component for providing high-quality healthcare services. ${ }^{6,7} \mathrm{PE}$ aims to support and strengthen patients' self-care responsibilities and abilities and then collaborate with their healthcare providers to achieve the maximum values of healthcare services and improve their health. ${ }^{8,9} \mathrm{PE}$ transforms the role of the patient in healthcare from a passive one (patient is a receiver) to an active one (patient is a partner). When interacting with the healthcare system, patients are supported in expressing their ideas and concerns to freely interact collaboratively with the healthcare provider in a process of shared decision-making towards, among other possible benefits, a better quality of life. ${ }^{10}$ Currently, strategies and policies that encourage $\mathrm{PE}$ in health and social care are gradually being established, mainly in developed Western countries. ${ }^{4,11,12}$ Governments are increasingly investing in research and programmes that encourage patients to be partners with healthcare professionals in taking responsibilities jointly to manage health. However, although the strategies and policies that support the engagement in improving the quality of healthcare services are political rhetoric in most countries, encouraging PE is still resisted by healthcare professionals. ${ }^{13}$ Queries, such as how to assess PE and how PE influences patients' quality of life, are rarely elaborated. Interest in PE stagnated, and the philosophy of health care never fundamentally changed.

The Hong Kong (HK) healthcare system is not yet ready to handle the fact that patients who are living with multiple chronic conditions do not truly need to have their diseases cured (impossible) but instead want a better life with the disease. ${ }^{14}$ The purpose of new healthcare services should transform from just helping people cope with the symptoms of illnesses or disabilities or regaining their best possible state of health to better managing their health and obtaining a better health-related quality of life (HRQoL). If the healthcare system is to effectively handle this transformation, its structure must evolve from the episode-driven "one-anddone" system to a system that is patient-centred with an integrated care approach. ${ }^{15}$ As such, patients' voices must be heard, and their needs and preferences must be considered and respected not only the treatment stage but at all stages (eg, diagnosis and rehabilitation) of the entire health care journey. Our healthcare system must be a system that emphasises value over volume. ${ }^{16}$
In $\mathrm{HK}$, the government encouraged using PE 10 years ago, ${ }^{17}$ though few empirical studies, which specifically emphasise the evaluation of PE in healthcare, support the policy makers' effort. Recently, increasing evidence has shown that PE is important for improving HRQoL, Boult et al., in US, identified that the promotion of PE is essential to successful care management programs targeting with improved quality of life, functional autonomy, and decreased hospital use. ${ }^{18}$ Dang-Vu et al preliminarily indicated that PE in health behaviours play a critical role in improving HRQoL and reducing healthcare utilization for patients living with chronic conditions. ${ }^{19}$ Barello et al developed a theoretical framework of PE based on psychological process and provided the empirical evidence in Italy that HRQoL deeply depends on the patient ability to engage in their care and the health expectation they have. ${ }^{20}$ However, studies to explore how the impact of PE on physical or psychological HRQoL has not yet been developed or widely reported in HK or China. ${ }^{10,21}$ The lack of evidence confirming such a relationship limits governments' willingness and determination to use $\mathrm{PE}$ as a potential driver to improve HRQoL, especially in primary care settings (eg, general outpatient clinics (GOPCs)). Of note, patients with chronic conditions need PE the most, and the healthcare system should enable a wide and equal delivery of services at first contact to improve HRQoL.

HK policy makers and public health researchers feel increasing pressure to develop strategies that effectively enable patients with chronic disease to actively engage in their healthcare to improve their HRQoL. The dilemma is not in overselling. According to the HK population projection, by 2034 , nearly $30 \%$ of its population will be 65 years and above, ${ }^{22}$ which will place a huge medical burden on the provision of healthcare services. HRQoL is increasingly accepted as a primary outcome of chronic disease studies that reflect the impact of diseases on patients' life, ${ }^{23}$ and PE is widely recognised as a powerful "weapon" for multiplying the healthcare system's ability to fight against chronic disease. ${ }^{24}$ The academic and political importance of this relationship must be studied, especially in primary care settings, to build a first-line defence to improve health outcome. Thus, the present study aims to assess PE and its correlation with HRQoL in the GOPCs in HK.

\section{Methods}

\section{Data collection}

A cross-sectional survey was conducted in one of the largest GOPCs in HK in 2017. In HK, GOPCs are committed to 
providing community-based primary care services for all the HK residents. The patients under the care of GOPCs are mainly chronic disease patients with stable conditions $(80 \%)$ and episodic disease patients with relatively mild symptoms. The inclusion criteria were as follows: (a) local language speaker (Cantonese), (b) aged $\geq 18$ years old, (c) visited the clinic in the last six months and (d) could understand the content of the interview. Moreover, we have gave priority to contact with chronically ill patients. A team of experienced investigators conducted the survey in the clinic for nearly two months. All of the interview arrangements and plans were fully discussed with and supported by the clinic's chief. The time slots, including both morning and afternoon sessions, were prepared after discussing with the chief nurse. All patients who satisfied the inclusion criteria and visited the appointed clinic during clinic hours were invited to participate in the interview. All patients who agreed to participate in the interview were provided some explanations of PE concepts and then asked to sign a written consent form where their rights, the purpose of the study and the collection procedure were fully stipulated. All patient information was kept confidential, except for the principle researcher. Ethical approval was obtained from the Joint Chinese University of Hong Kong-New Territories East Cluster Clinical Research Ethics Committee.

\section{Study instruments}

Patient engagement index (PEI)

The Patient Engagement Index (PEI, traditional Chinese version) was developed in HK to specifically evaluate the patients' performance on engaged relationships with healthcare professionals in the outpatient setting at four different stages of health care. PEI has been proved has good reliability (Cronbach's alpha $=0.92$ ) and validity. The PEI has 20 items and assesses the level of PE based on patient's self-experience at stages of diagnosis, treatment, rehabilitation and health maintenance. Each stage has five items to assess the different aspects (levels) of patientprofessional interaction (self-search information, enquiry, explanation, discussion and shared decision-making). Each item of PEI was assigned a specific weight to reflect its importance in PE. After transformation, the overall score of PEI ranges from 0 to 100 , the higher the score is, the better the PE is. The PEI was developed while fully considering the international experience and the local patients' needs and is a scientific instrument that was first developed and applied in HK to assess patient engagement. ${ }^{25}$

\section{EQ-5D-5L}

EQ-5D-5L, developed by EuroQol, is simple, generic and standardised for measuring an individual's health status. ${ }^{26}$ EQ-5D-5L has two parts, the first is a descriptive system contains five dimensions, including mobility, self-care, usual activities, pain/discomfort and anxiety/depression, ${ }^{10}$ There are five levels within each dimension, from "no problems" to "extreme problems," which lead to 3,125 possible health statuses. It provides a simple descriptive profile and a single index value for comparing the health status of the population. ${ }^{27}$ The second part is the EQ visual analogue scale (EQ VAS). EQ VAS records the respondent's self-rated health on a vertical scale where the endpoints are labelled "Best imaginable health state" and "Worst imaginable health state". ${ }^{10}$ The reported health states of five dimensions can be converted into a single health index (utility score) using a scoring algorithm based on cultural health preferences. The utility score ranges between 0 and 1 , where 1 represents "full health," 0 represents death, and negative values represent health states worse than death. The HK value set of EQ-5D-5L was developed by Wong in 2016, following the new international EQ-5D-5L valuation protocol. ${ }^{28}$ The mean index value for the general population in HK was 0.9186. The visual analogue rating scale (VAS) was not included in our current study.

\section{Data analysis}

$\mathrm{R}$ ( $\mathrm{R}$ foundation, version 3.5.0) was used for data analysis. Descriptive statistics were generated for the patients' socio-economic characteristics (ie, gender, age, educational level, marriage status, living status, working status, chronic conditions, status for receiving government allowance and having a caregiver or not), PEI score and EQ-5D5L utility. ANOVA and ANCOVA F-tests with bootstrap were applied to examine the between-group differences based on these variables. ${ }^{10}$ In this study, the statistical significance was set to $p$-value $<0.05$.

Robust linear regression models were built in this study for each process using the $\mathrm{M}$ estimator. ${ }^{29}$ In the analysis, we examined the relationship between PE using the PEI and HRQoL using the EQ-5D utility. The PEI score was set as the dependent variable (DV), and the EQ-5D utility was set as the independent variable (IV). We also examined whether this relationship varied by adjusting a series of socio-economic characteristics. Therefore, three models were used in this study: (1) the PE-HRQoL model, which 
examined the relationship between PE and HRQoL (EQ5D utility as the IV), (2) the PE-HRQoL-D model, which examined the relationship between PE and HRQoL adjusted by demographic chrematistics (EQ-5D utility, gender, age and educational level as the IVs), and (3) a full model, which examined the relationship between PE and HRQoL adjusted by all the socio-economic variables (EQ-5D utility, gender, age, educational level, government allowance, marriage status, working status, living status, chronic conditions and having a caregiver or not as the IVs).

\section{Results}

A total of 686 patients (response rate $=64.1 \%$ ) completed the questionnaire. Among them, 56\% were female, nearly $50 \%$ were older than 55 and more than $60 \%$ reached at least a post-secondary educational level or above. Moreover, $91.1 \%$ of the respondents were not government allowance receivers, $53.6 \%$ had fulltime jobs and $71.4 \%$ reported to have at least one type of chronic condition (Table 1). Compared with the general population, except for age, our sample showed no other significant differences (https://www.bycensus2016.gov.hk/en/).

Figure 1 indicates the distribution of the EQ-5D utility and the PEI score. The distribution of the EQ-5D utility was negatively skewed with a clustering at 1.0. The patients showed a high possibility of reporting the utility of full health, and most of the utility were reported greater than 0.7. The histogram of PEI indicated that the overall distribution of PEI score was nearly normal but slightly positively skewed. Most respondents scored between 25 and 50. Figure 2 indicated that participants with higher educational level were prone to show better HRQoL for both male and female. For participants with secondary educational level, the mean PEI scores for male and female were similar, however, the range of EQ-5D utility for female $(0.0 \sim 1.0)$ was much wider than male's $(0.6 \sim 1.0)$.

Table 2 presents the PEI scores, the EQ-5D utility and VAS based on the background characteristics of the sample. The overall mean EQ-5D utility was $0.92(0-1.0)$. The males (mean $=0.93, \mathrm{SD}=0.12$ ) obtained a higher utility than the females, and the respondents with the highest educational level also had the best performance (utility $=0.93$, SD $=0.09$ ). The mean VAS score was 73.26 , sd was 14.08. For PE, the mean (SD) PEI score was 37.02 (16.14). The respondents (PEI score) who were female (38.03), younger (45.87), highly educated (45.31), single
(41.53), and had no chronic conditions (40.07) had a positive correlation with a better PE (Table 2).

Table 3 indicates the mean score of the PEI for each dimension of the EQ-5D index at different stages of health care. Patients reported having more problems at mobility, self-care and usual activities tend to report a higher PEI score at all four stages of health care. For pain/discomfort, patients with moderate problem tends to have more positive attitude toward engagement into healthcare. However, for anxiety/depression, patients with no or slight mental problems tend to be more engaged.

In the regression model, we found that the PEI score increased significantly with the increment of the EQ-5D utility (coefficient $=2.66, \mathrm{SE}=5.11$ ). After adjustment by other demographic characteristics in Model 2, the correlation between PE and utility was weakened (coefficient $=1.77, \mathrm{SE}=4.64$ ) but still positive and significant. All of the demographic variables, such as gender, age and education, showed a significant relationship with PE. However, for Model 3 (full-model), after applying all of the socio-economic variables in the model, the relationship between PE and utility became weaker (coefficient $=1.04$, $\mathrm{SE}=4.69)$ and insignificant. Compared with that in Model 2 , the other newly added variables also did not reach statistical significance (Table 4).

\section{Discussion}

\section{Key findings of this study}

Our study is the first to use a locally valid and reliable instrument for measuring PE to provide empirical evidence to reveal the level of patient engagement in public healthcare system in HK. A mean PEI score of 37.02 was reported in the survey. The results of synonymous patient experience studies in other Western countries are as follows: the Dutch mean was 61.3, the American mean was 61.9 and the Danish mean was $64.2^{30}$ using the patient activation measure (an instrument developed in the US to assess patients' activation in health care, 0-100), where the performance of local patient engagement is not optimistic. (Note: The consideration of the comparison must be taken cautiously as they are two different concepts being validated in different countries). Moreover, our results also serve as the baseline for future assessment of $\mathrm{PE}$ in different settings or for different health conditions. For EQ-5D, the mean utility in the GOPC shows no difference from the HK general population. ${ }^{10}$ 
Table I Demographics of respondents in the survey and comparison with the general population

\begin{tabular}{|c|c|c|c|c|}
\hline & \multicolumn{2}{|c|}{ Participant } & \multirow{2}{*}{$\begin{array}{l}\text { General population } \\
\%\end{array}$} & \multirow[t]{2}{*}{$p$-value } \\
\hline & $\mathbf{n}$ & $\%$ & & \\
\hline \multicolumn{5}{|l|}{ Gender } \\
\hline Male & 302 & 44.0 & 46.1 & 0.89 \\
\hline Female & 384 & 56.0 & 53.9 & \\
\hline \multicolumn{5}{|l|}{ Age group (18-80) } \\
\hline $18-34$ & 142 & 20.7 & 37.4 & 0.03 \\
\hline $35-44$ & 88 & 12.8 & 15.6 & \\
\hline $45-54$ & 149 & 21.7 & 16.8 & \\
\hline $55-64$ & 210 & 30.6 & 14.8 & \\
\hline$>65$ & 97 & 14.1 & 15.3 & \\
\hline \multicolumn{5}{|l|}{ Education } \\
\hline No education/ Primary & 99 & 14.4 & 18.9 & 0.47 \\
\hline Secondary & 152 & 22.2 & 50.3 & \\
\hline Post-secondary & 243 & 35.4 & & \\
\hline Tertiary or above & 192 & 28.0 & 30.8 & \\
\hline \multicolumn{5}{|c|}{ Receive government allowance } \\
\hline Yes & 61 & 8.9 & II.I & 0.81 \\
\hline No & 625 & 91.1 & 88.9 & \\
\hline \multicolumn{5}{|l|}{ Current living status } \\
\hline Live alone & 56 & 8.2 & & \\
\hline Live with family/ others & 627 & 91.4 & & \\
\hline Live in institution ${ }^{2}$ & 3 & 0.4 & & \\
\hline \multicolumn{5}{|l|}{ Marriage } \\
\hline Single & 177 & 25.8 & 30.1 & 0.46 \\
\hline Married & 467 & 68.1 & 58.4 & \\
\hline Divorce & 19 & 2.8 & 4.6 & \\
\hline Widow & 23 & 3.4 & 6.4 & \\
\hline \multicolumn{5}{|l|}{ Current working status } \\
\hline Retired & 124 & 18.1 & & \\
\hline Unemployment & 25 & 3.6 & & \\
\hline Employment & 368 & 53.6 & 51.3 & \\
\hline Housewife & 135 & 19.7 & & \\
\hline Full time student & 34 & 5.0 & & \\
\hline \multicolumn{5}{|c|}{ Having chronic condition ${ }^{\#}$} \\
\hline Yes & 492 & 71.4 & & \\
\hline No & 188 & 27.1 & & \\
\hline \multicolumn{5}{|l|}{ Caregiver } \\
\hline Yes & 28 & 4.1 & & \\
\hline No & 658 & 95.9 & & \\
\hline
\end{tabular}

Note: ${ }^{\#}$ people reported uncertain.

Second, a high-performing healthcare system should provide services to improve the health of its population. ${ }^{31}$ $\mathrm{PE}$ is an effective way to connect patients with their healthcare providers to improve health outcomes. ${ }^{32}$ In our study, people with different socio-economic backgrounds showed, to some extent, different performance on PE where the socially vulnerable population seldom engaged in health care. Although we know that engaging patients in healthcare is not an easy task, our study provided valuable and evidence-based lessons that no one-size-fits-all strategy 


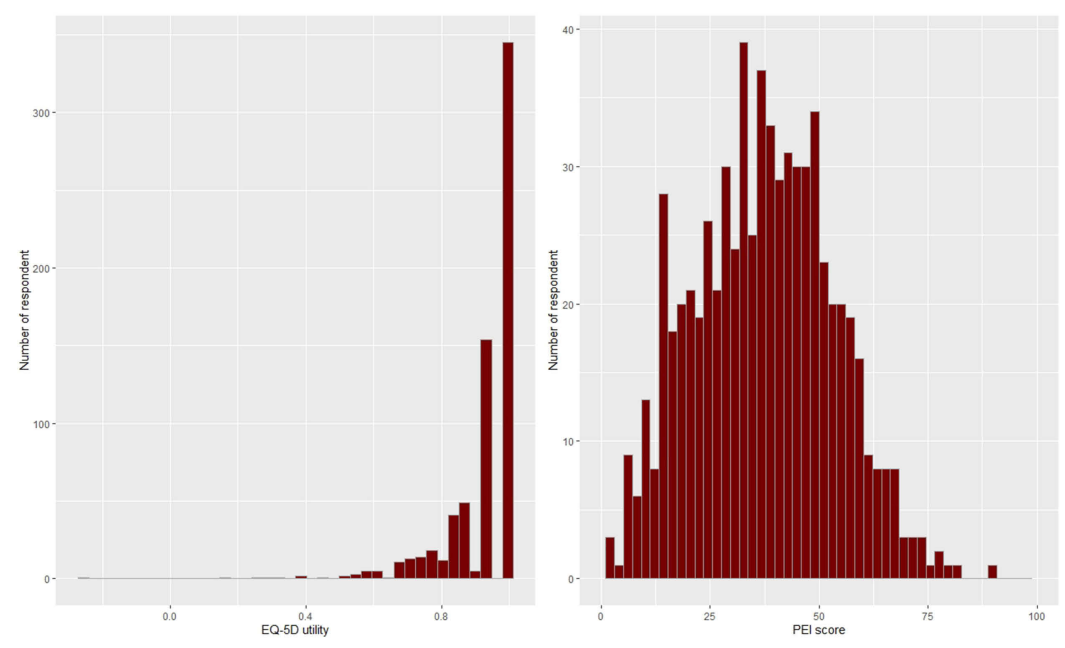

Figure I The distribution of PEI score and EQ-5D utility.

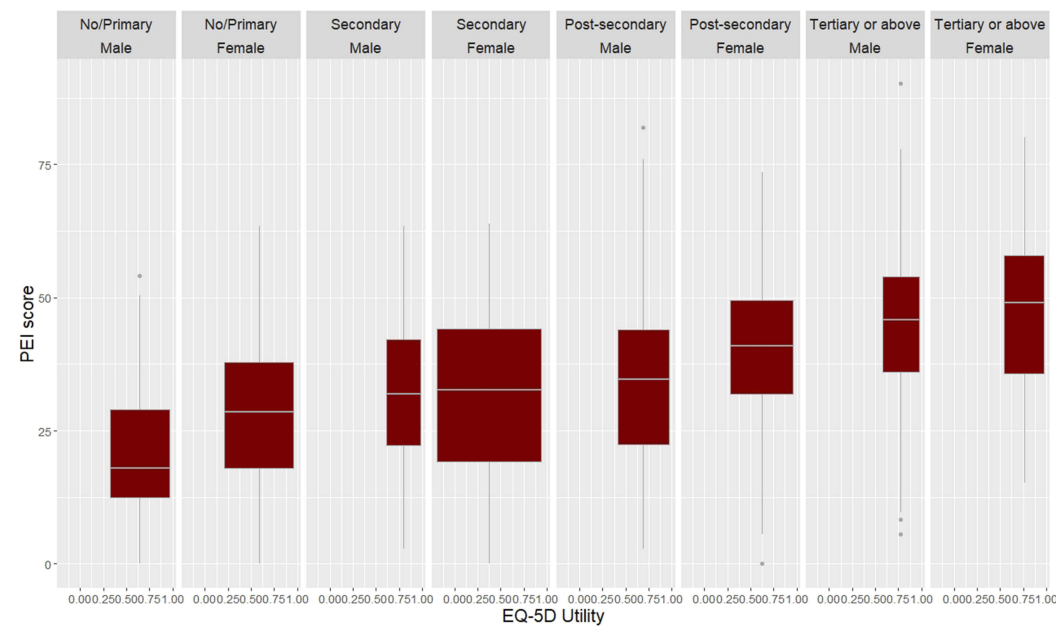

Figure 2 The boxplot of PEI score and EQ-5D utility based on sex and educational level.

could rapidly improve patient engagement. If we want to improve the patient's quality of life through encouraging engagement, their background characteristics must be preferentially considered in providing healthcare services.

Lastly and importantly, our study explores the potential relationship between PE and HRQoL in the GOPCs in HK. Essentially and academically, there is a preliminary agreement that PE could positively affect HRQoL for some specific diseases, ${ }^{12}$ however, the association is not clear, and no agenda for future studies exists. Our study initiates a good attempt at filling this gap, which empirically confirms some previous assumptions and hypotheses demonstrating that encouraging patients to engage in their healthcare could improve their HRQoL in primary care. However, we also found that this PE-HRQoL relationship is inconsistent and varied when considering background characteristics and different stages of healthcare, and thus more efforts will be needed in the future for more a substantial exploration.

\section{What is already known on this topic}

$\mathrm{PE}$ has recently been recognised as one of most valuable tools in the healthcare provider's arsenal for improving patient-centred care (PCC) for dealing with the increased epidemic of chronic diseases. Both PE and PPC are patient-centric and thus have natural connections. In the PCC pattern, any single patient should be treated as a unique individual, and his/her health needs or preferred outcomes are the main driving force behind all decisions made by professionals. Coulter indicated that professionals should engage patients in health care, not only about 
Table 2 The PEI score and EQ-5D utility based on demographic characteristics

\begin{tabular}{|c|c|c|c|c|c|c|c|c|c|}
\hline & \multicolumn{3}{|c|}{ EQ-5D utility } & \multicolumn{3}{|c|}{ EQ-5D VAS } & \multicolumn{3}{|c|}{ PEI score } \\
\hline & Mean & sd & $p$-value & Mean & sd & $p$-value & Mean & sd & $p$-value \\
\hline Overall & 0.92 & 0.13 & & 73.26 & 14.08 & & 37.02 & 16.14 & \\
\hline \multicolumn{10}{|l|}{ Gender } \\
\hline Male & 0.93 & 0.12 & $<0.01$ & 73.28 & 13.85 & 0.97 & 32.75 & 16.60 & $<0.05$ \\
\hline Female & 0.91 & 0.13 & & 73.24 & 14.27 & & 38.03 & 15.72 & \\
\hline \multicolumn{10}{|l|}{ Age group (18-80) } \\
\hline $18-34$ & 0.93 & 0.14 & 0.78 & 73.07 & 15.05 & 0.98 & 45.87 & 15.0 & $<0.001$ \\
\hline $35-44$ & 0.91 & 0.14 & & 72.90 & 15.18 & & 40.63 & 15.89 & \\
\hline $45-54$ & 0.92 & 0.13 & & 73.79 & 14.52 & & 37.59 & 15.17 & \\
\hline $55-64$ & 0.92 & 0.10 & & 73.12 & 12.84 & & 33.83 & 15.04 & \\
\hline$>65$ & 0.91 & 0.14 & & 73.35 & 12.73 & & 26.84 & 14.04 & \\
\hline \multicolumn{10}{|l|}{ Education } \\
\hline No education/ Primary & 0.90 & 0.15 & $<0.05$ & 72.58 & 15.23 & 0.99 & 25.72 & 14.06 & $<0.001$ \\
\hline Secondary & 0.91 & 0.15 & & 73.39 & 12.94 & & 32.37 & 15.12 & \\
\hline Post-secondary & 0.92 & 0.12 & & 73.93 & 13.32 & & 38.06 & 14.49 & \\
\hline Tertiary or above & 0.93 & 0.09 & & 72.66 & 15.28 & & 45.31 & 15.22 & \\
\hline \multicolumn{10}{|l|}{ Receive government allowance } \\
\hline Yes & 0.86 & 0.16 & $<0.05$ & 71.23 & 17.05 & 0.32 & 28.79 & 15.44 & $<0.001$ \\
\hline No & 0.92 & 0.12 & & 73.46 & 13.75 & & 37.83 & 15.99 & \\
\hline \multicolumn{10}{|l|}{ Current living status } \\
\hline Live alone & 0.91 & 0.12 & 0.79 & 73.66 & 12.56 & 0.82 & 31.74 & 13.82 & $<0.01$ \\
\hline Live with family/ others & 0.92 & 0.13 & & 73.26 & 14.22 & & 37.47 & 16.23 & \\
\hline \multicolumn{10}{|l|}{ Marriage } \\
\hline Single & 0.93 & 0.09 & 0.71 & 71.98 & 14.48 & 0.29 & 41.53 & 16.27 & $<0.01$ \\
\hline Married & 0.92 & 0.14 & & 73.74 & 14.11 & & 35.85 & $|5.9|$ & \\
\hline Divorce & 0.91 & 0.11 & & 72.63 & 13.16 & & 34.09 & $|2.5|$ & \\
\hline Widow & 0.90 & 0.13 & & 73.91 & 10.66 & & 28.62 & $15.2 \mid$ & \\
\hline \multicolumn{10}{|l|}{ Current working status } \\
\hline Retired & 0.92 & 0.13 & 0.25 & 75.04 & 13.23 & 0.38 & 29.86 & $|5.3|$ & $<0.001$ \\
\hline Unemployment & 0.91 & 0.10 & & 69.80 & 16.92 & & 34.30 & 18.48 & \\
\hline Employment & 0.92 & 0.11 & & 73.41 & 13.89 & & 39.27 & 15.67 & \\
\hline Housewife & 0.90 & 0.17 & & 71.44 & 14.52 & & 36.41 & 15.75 & \\
\hline Full time student & 0.93 & 0.11 & & 74.88 & 14.56 & & 43.33 & 15.76 & \\
\hline \multicolumn{10}{|l|}{ Having chronic condition } \\
\hline Yes & 0.92 & 0.13 & 0.39 & 73.13 & 13.89 & 0.56 & 35.97 & 16.31 & $<0.01$ \\
\hline No & 0.92 & 0.10 & & 73.83 & 14.38 & & 40.07 & 15.37 & \\
\hline \multicolumn{10}{|l|}{ Caregiver } \\
\hline Yes & 0.85 & 0.24 & 0.59 & 74.64 & $|5.5|$ & 0.63 & 38.08 & 24.73 & 0.89 \\
\hline No & 0.92 & 0.12 & & 73.20 & 14.02 & & 36.89 & 15.70 & \\
\hline
\end{tabular}

clinical decisions, but about patients' emotional, mental and spiritual preferences. ${ }^{33}$ However, in recent decades, although PE has been chosen as a top candidate for achieving PPC, evaluating PE and considering the different political and cultural realities in different countries is still underdeveloped, thereby causing a series of bias, disputations and uncertainties for both the government and public to embrace PE.

Furthermore, a growing international interest in PE exists in healthcare services to fight against chronic diseases 
Table 3 The mean score of PEI of different dimensions on different levels of EQ-5D dimension

\begin{tabular}{|c|c|c|c|c|c|c|c|c|c|c|c|}
\hline \multirow[t]{2}{*}{ EQ-5D } & \multirow[t]{2}{*}{$\mathbf{n}$} & \multicolumn{2}{|c|}{ Diagnosis } & \multicolumn{2}{|c|}{ Treatment } & \multicolumn{2}{|c|}{ Rehabilitation } & \multicolumn{2}{|c|}{ Health Maintenance } & \multicolumn{2}{|c|}{ Overall } \\
\hline & & Mean & sd & Mean & sd & Mean & sd & Mean & sd & Mean & sd \\
\hline \multicolumn{12}{|l|}{ Mobility } \\
\hline I & 621 & 42.36 & 22.49 & 45.9 & 17.0 & 22.91 & 20.61 & 24.45 & 18.62 & 37.06 & 16.22 \\
\hline 2 & 46 & 37.14 & 21.78 & 45.2 & 13.15 & 24.52 & 20.10 & 24.67 & 16.91 & 35.62 & 14.09 \\
\hline 3 & 17 & 42.65 & 26.5 & 49.51 & 16.26 & 25.33 & 22.39 & 23.53 & 16.75 & 39.16 & 19.61 \\
\hline 4 & 2 & 50.0 & 10.0 & 39.58 & 14.73 & 31.25 & 18.66 & 30.0 & 24.65 & 40.28 & 11.13 \\
\hline \multicolumn{12}{|l|}{ Self-care } \\
\hline 1 & 675 & 42.02 & 22.52 & 45.85 & 16.75 & 22.93 & 20.48 & 24.50 & 18.50 & 36.93 & 16.11 \\
\hline 2 & 8 & 35.42 & 21.25 & 46.88 & 13.5 & 31.08 & 22.48 & 20.62 & $17.6 \mid$ & 37.79 & 15.64 \\
\hline 3 & 3 & 63.89 & 19.25 & 61.11 & 17.35 & 40.74 & 34.42 & 25.0 & 18.03 & 55.25 & 18.75 \\
\hline \multicolumn{12}{|l|}{ Usual activities } \\
\hline I & 661 & 41.82 & 22.44 & 45.81 & 16.73 & 22.96 & 20.44 & 24.58 & 18.56 & 36.86 & 16.03 \\
\hline 2 & 20 & 44.17 & 23.43 & 47.50 & 16.74 & 23.06 & 23.60 & 21.75 & 16.64 & 38.24 & 17.82 \\
\hline 3 & 5 & 66.67 & 26.35 & 60.42 & 14.23 & 48.96 & 20.01 & 23.75 & 14.93 & 58.68 & 17.71 \\
\hline \multicolumn{12}{|l|}{ Pain/discomfort } \\
\hline I & 393 & 41.92 & 23.01 & 45.64 & $|7.5|$ & 23.67 & 20.99 & 24.58 & $|8.6|$ & 37.08 & 16.63 \\
\hline 2 & 191 & 43.94 & $20.4 I$ & 47.36 & 16.04 & 23.98 & 20.37 & 25.16 & 18.74 & 38.43 & $15.4 \mid$ \\
\hline 3 & 96 & 39.15 & 23.97 & 45.27 & 14.35 & 19.18 & 19.30 & 22.86 & 16.99 & 34.53 & 15.24 \\
\hline 4 & 6 & 36.11 & 30.12 & 29.17 & 13.69 & 20.60 & 16.70 & 20.0 & 15.5 & 28.63 & 17.23 \\
\hline \multicolumn{12}{|l|}{ Anxiety/depression } \\
\hline I & 549 & 42.14 & 22.13 & 45.73 & 16.81 & 23.41 & 20.71 & 25.02 & 18.40 & 37.1 & 16.03 \\
\hline 2 & 109 & 42.43 & 22.70 & 46.75 & 16.14 & 22.52 & 20.72 & 23.30 & 18.70 & 37.23 & 16.4 \\
\hline 3 & 21 & 40.48 & 30.31 & 48.41 & 18.42 & 18.39 & 18.03 & 18.33 & 18.12 & 35.76 & 17.87 \\
\hline 4 & 7 & 33.33 & 27.64 & 40.48 & 15.54 & 16.85 & 14.23 & 17.14 & 15.76 & 31.94 & 18.27 \\
\hline
\end{tabular}

in which the evidence of the impact of PE on HRQoL is increasing but fragmented. In 2013, the International Society for Quality of Life research (ISOQOL) hosted the first symposium to officially explore the potential of PE in the field of QoL. ${ }^{12}$ In the symposia, researchers arrived at a consensus that a framework or toolkit for assessing PE within HRQL research should be one of the directions of future studies. However, the PE-HRQoL relationship remains complicated. Thus, we need more studies that look into the complexity of this association and consider the cultural-specific aspects.

\section{What has been added by our study}

Some important findings related to PE were found in this study. Previous research has indicated that PE could be perceived as a culturally-sensitive concept that is an expression of patients' subjective feelings influenced by the objective healthcare system with which they are in contact. ${ }^{6,34}$ However, we found that the concept of culture is more complicated than previous studies assumed.
Besides the organisational culture in the healthcare system, the social culture may also influence PE to some extent. In this study, females had a lower utility but had better PE results. In Chinese culture, females are supposed to play the role of family caretaker. ${ }^{35}$ In our study, the findings might indicate that the female participants who proactively engaged in health care are, mostly, not engaging for themselves but for their families. Their highly engaged attitude did not result in maximising their own utility. Moreover, although previous literature has pointed prominently that education is one of the most important determinants to explain the health disparities, ${ }^{36}$ our study indicated that education was not only critical to people's attitude toward health care but also has a profound impact on PE. We believe educational inequity not only affect the people's health outcome, but affect their ability to navigate the system and further decrease their willingness to be involved in health care. As Field indicated in 1976, that health systems are integral components of the social system, which perform a series of functions and in turn 
Table 4 Regression analyses of patient engagement and EQ-5D and all socio-demographic variables

\begin{tabular}{|c|c|c|c|c|c|c|c|c|c|}
\hline & \multicolumn{3}{|l|}{ Model I } & \multicolumn{3}{|l|}{ Model 2} & \multicolumn{3}{|l|}{ Model 3} \\
\hline & Coefficient & $\begin{array}{l}95 \% \\
\text { Confidence } \\
\text { Interval }\end{array}$ & SE & Coefficient & $\begin{array}{l}95 \% \\
\text { Confidence } \\
\text { Interval }\end{array}$ & SE & Coefficient & $\begin{array}{l}95 \% \\
\text { Confidence } \\
\text { Interval }\end{array}$ & SE \\
\hline $\begin{array}{l}\text { EQ-5D utility } \\
\text { Female } \\
35-44 \\
45-54 \\
55-64 \\
>65 \\
\text { Secondary } \\
\text { Post-secondary } \\
\text { Tertiary or } \\
\text { above } \\
\text { Receive } \\
\text { allowance } \\
\text { Live with family/ } \\
\text { others } \\
\text { Married } \\
\text { Divorce } \\
\text { Widow } \\
\text { Unemployment } \\
\text { Employment } \\
\text { Housewife } \\
\text { Full time student } \\
\text { No chronic } \\
\text { condition } \\
\text { Have caregiver }\end{array}$ & $2.66 *$ & $0.23 \sim 12.31$ & 5.11 & $\begin{array}{l}1.77^{*} \\
3.42^{*} \\
-2.13 \\
-4.48^{*} \\
-6.07^{* *} \\
-11.92^{* * *} \\
5.13^{*} \\
9.82^{* * *} \\
15.39 * * *\end{array}$ & $\begin{array}{l}0.55 \sim 8.87 \\
1.11 \sim 5.73 \\
-6.54 \sim 1.93 \\
-8.33 \sim-0.63 \\
-9.87 \sim-2.27 \\
-16.38 \sim-7.46 \\
1.21 \sim 9.06 \\
6.08 \sim 13.56 \\
11.09 \sim 19.68\end{array}$ & $\begin{array}{l}4.64 \\
1.18 \\
2.16 \\
1.97 \\
1.94 \\
2.28 \\
2.00 \\
1.91 \\
2.19\end{array}$ & $\begin{array}{l}1.04 \\
3.74 * \\
-3.46 \\
-5.88^{*} \\
-7.49 * * \\
-13.95 * * * \\
4.76 * \\
9.39 * * * \\
14.97 * * * \\
\\
2.06 \\
1.04 \\
1.46 \\
-0.86 \\
-4.19 \\
-3.09 \\
-1.56 \\
-1.66 \\
-1.15 \\
-0.75 \\
2.09\end{array}$ & $\begin{array}{l}-10.09 \sim 8.29 \\
1.15 \sim 6.32 \\
-8.21 \sim 1.29 \\
-10.4 \sim-1.35 \\
-12.2 \sim-2.78 \\
-19.94 \sim-7.96 \\
0.77 \sim 8.75 \\
5.54 \sim 13.25 \\
10.53 \sim 19.41 \\
-2.77 \sim 6.9 \\
-2.57 \sim 4.66 \\
-6.46 \sim 9.38 \\
-8.05 \sim 6.32 \\
-11.33 \sim 2.95 \\
-10.03 \sim 3.84 \\
-6.33 \sim 3.01 \\
-5.24 \sim 2.93 \\
-6.01 \sim 2.89 \\
-3.49 \sim 1.99 \\
-3.87 \sim 8.06\end{array}$ & $\begin{array}{l}4.69 \\
1.32 \\
2.42 \\
2.31 \\
2.41 \\
3.06 \\
2.03 \\
1.97 \\
2.27 \\
\\
2.47 \\
\\
1.84 \\
\\
4.04 \\
3.67 \\
3.64 \\
3.54 \\
2.38 \\
2.09 \\
2.27 \\
1.40 \\
\\
3.05\end{array}$ \\
\hline
\end{tabular}

Notes: $* p<0.05, * * p<0.01, * * * p<0.001$

Abbreviation: SE, Standard error.

receive support and resources. ${ }^{37}$ Improving PE requires diminishing socially cultural bias and encouraging collaborations among different sub-systems, instead of focusing only on the healthcare system. Boutin has also suggested that culture and process change is a key strategy to facilitate the implementation of PE. ${ }^{38}$

Although there is an agreement of the importance of PE in bringing benefits to HRQoL, ${ }^{12,39}$ the current evidence is generally non-systematic, sporadic and inconsistent. This study found that the relationship between PE and HRQoL is positive but varied significantly when considering the respondents' different socio-economic characteristics. Although previous empirical literature indicated that there are striking disparities in health by socioeconomic status, ${ }^{40,41}$ the varied relationship between HRQoL and PE was rarely empirically explored. Our study indicated that there is rarely one PE improvement intervention that can maximise all of the patients' utility or one intervention that can maximise all of one patient's utility. Much remains to be discovered and evaluated to achieve a meaningful point of balancing the PE-HRQoL relationship for all the patients, especially, the vulnerable population, eg old, low educated or live with chronic conditions. Le V Hoi et al indicated that the involvement of vulnerable population in social and health care interventions for improving HRQoL in a context with limited resources is challenging but possible. ${ }^{42}$ Developing an effective and innovative policy must value patients' preferences and needs and consider social equity, which requires a willingness to admit that there is no-one-size-fits-all strategy to improve HRQoL through improving PE. For example, eHealth is a cost-effective way of training the young or highly educated public and patients to have more knowledge or skills about their diseases or bodies. ${ }^{43}$ However, online information and abandoning traditional methods, such as television, pamphlets or telephone, might lead to larger barriers 
for elderly or impecunious people. A substantial disadvantage is that using an inappropriate strategy may inadvertently distance the patients from the healthcare system, potentially leading to some health risks, an unhealthy lifestyle and a lower quality of life. ${ }^{44}$ As Haywood indicated, we must embrace these challenges with flexibility, honesty and openness. ${ }^{12}$

Furthermore, based on the analysis of the EQ-5D index, our results demonstrate that even patients with severe problems regarding mobility, self-care and usual activities still eagerly engage in health care management. Conversely, those patients experiencing pain and depression tend to be less engaged with the increasing problems. It seems the physical inconvenience did not disrupt the patients' willingness to engage the healthcare system, but psychological discomfort did and substantially influenced their ability to engage in health care. This pattern is interesting but reasonable. Physical disability can encourage patients to be more engaged to enhance recovery, but mental illness can affect the way a patient thinks, feels and interacts with society. To our knowledge, no studies have directly reported the PE-HRQoL relationship using EQ-5D measurement. Even considering the other synonymous concepts, such as activation, the finding is still conflicted. Shortell indicated a positive relationship between patient activation and both physical and psychological functions. ${ }^{45}$ However, Aung found no systematic relationship between the EQ-5D index and patient activation. ${ }^{46}$ Therefore, in future studies, we suggest HRQoL measurements should be routinely embedded in PE studies, which could strengthen our understanding of patients' special needs in health care and help us transfer our culture and focus of PE improvement to conceptualise benefits based on patients' diseases, symptoms or preferences at different stages of their journey to improving health.

This study has several limitations. First, this study is a cross-sectional study, and the causal relationship between PE and HRQoL might be not derived. Second, due to the research purpose, all of the participants are from GOPCs and thus some problems may exist when generalising the results to patients from other settings. Moreover, no convergent validity has been tested during the development of the PEI. Therefore, the findings of this study may have some limitations on generalisability in other countries or regions. Lastly, more than $30 \%$ of the patients refused to participate in our study in the GOPC, and the absence of comparison between respondents and non-respondents might cause some selection bias.

\section{Conclusion}

Our empirical study indicated a direct relationship between patient engagement and HRQoL in HK. The findings presented that patients who scored high in the PEI tend to have a high EQ-5D utility. However, when adjusting according to different socio-economic factors, the relationship is weakened and even insignificant, indicating that such a relationship might be deeply influenced by an individual's physical, psychological or social characteristics. These findings indicate that no one-size-fits-all strategy to improve HRQoL through engaging patients in health care exists. Therefore, more customised and tailored plans that focus on the specific needs and preferences of individual patients should be designed and implemented. The findings of our cross-sectional study are limited. The potentials of the relationship between PE and different aspects of HRQoL require more substantial explorations and methodological developments in different settings of the healthcare system.

\section{Data sharing statement}

No additional data are available.

\section{Acknowledgment}

The authors received no specific funding for this work.

\section{Author contributions}

All authors contributed to data analysis, drafting or revising the article, gave final approval of the version to be published, and agree to be accountable for all aspects of the work.

\section{Disclosure}

The authors report no conflicts of interest in this work.

\section{References}

1. Lutz W, Sanderson W, Scherbov S. The coming acceleration of global population ageing. Nature. 2008;451:716-719. doi:10.1038/ nature 06516

2. Stewart M. Towards a global definition of patient centred care. The patient should be the judge of patient centred care. BMJ. 2001;322:444-445. doi:10.1136/bmj.322.7284.444

3. Epstein RM, Street RL. The value of patient-centered care. Patient Educ Couns. 2007;68:179-185.

4. Coulter A. Patient engagement-what works? J Ambul Care Manage. 2012;35:80-89. doi:10.1097/JAC.0b013e318249e0fd 
5. Yu W, Li M, Ye F, Xue C, Zhang L. Patient preference and choice of healthcare providers in Shanghai, China: a cross-sectional study. BMJ Open. 2017;(7). doi:10.1136/bmjopen-2017-016418

6. Barello S, Graffigna G, Vegni E. Patient engagement as an emerging challenge for healthcare services: mapping the literature. Nurs Res Pract. 2012;2012:905934. doi:10.1155/2012/372617

7. Forbat L, Cayless S, Knighting K, Cornwell J, Kearney N. Engaging patients in health care: An empirical study of the role of engagement on attitudes and action. Patient Educ Couns. 2009;74:84-90. doi:10.1016/j.pec.2008.07.055

8. Vahdat S, Hamzehgardeshi L, Hessam S, Hamzehgardeshi Z. Patient involvement in health care decision making: a review. Iran Red Crescent Med J. 2014;16:e12454. doi:10.5812/ircmj.12454

9. Pomey M-P, Ghadiri DP, Karazivan P, Fernandez N, Clavel N. Patients as partners: a qualitative study of patients' engagement in their health care. PLoS One. 2015;10:e0122499. doi:10.1371/journal. pone. 0122499

10. Xu RH, Cheung AAWL, Wong ELY. The relationship between shared decision-making and health-related quality of life among patients in Hong Kong SAR, China. Int J Qual Heal Care. 2017;29:534-540. doi:10.1093/intqhe/mzx067

11. Laurance J, Henderson S, Howitt PJ, et al. Patient engagement: four case studies that highlight the potential for improved health outcomes and reduced costs. Health Aff. 2014;33:1627-1634. doi:10.1377/ hlthaff. 2014.0375

12. Haywood K, Brett J, Salek S, et al. Patient and public engagement in health-related quality of life and patient-reported outcomes research: what is important and why should we care? Findings from the first ISOQOL patient engagement symposium. Qual Life Res. 2015;24:1069-1076. doi:10.1007/s11136-014-0796-3

13. Skillman M, Cross-Barnet C, Singer RF, et al. Physician engagement strategies in care coordination: findings from the Centers for Medicare \& Medicaid Services' Health Care Innovation Awards Program. Health Serv Res. 2017;52:291-312. doi:10.1111/1475-6773.12582

14. Groves T, Wagner EH. High quality care for people with chronic diseases. BMJ. 2005;330:609-610. doi:10.1136/bmj.330.7492.609

15. Beveridge B Chronic conditions treatment is not "one and done"; 2018. Available from: https://catalyst.nejm.org/chronic-conditionstreatment-not-done/. Accessed August 16, 2019.

16. Chad Mather RC, Bozic KJ. Value-based care. J Shoulder Elb Surg. 2013;22:1599-1600. doi:10.1016/j.jse.2013.10.007

17. Hospital Authority. Helping People Stay Healthy - Strategic Service Plan 2009-1012; 2009. Available from: http://www.ha.org.hk/ho/ corpcomm/Strategic\%20Plan/2012-17.pdf. Accessed August 19, 2019.

18. Boult C, Green AF, Boult LB, Pacala JT, Snyder C, Leff B. Successful models of comprehensive care for older adults with chronic conditions: evidence for the Institute of Medicine's "Retooling for an aging America" report. $J$ Am Geriatr Soc. 2009;57:2328-2337. doi:10.1111/jgs.2009.57.issue-12

19. Dang-Vu G, Rein L, Berens B, Szabo A, Venkatesan T. Patient engagement is associated with health related quality of life in adults with cyclic vomiting syndrome. Gastroenterology. 2017;152:S117S117. doi:10.1016/S0016-5085(17)30729-1

20. Barello S, Graffigna G. Engaging patients to recover life projectuality: an Italian cross-disease framework. Qual Life Res. 2015;24:1087-1096. doi:10.1007/s11136-014-0846-x

21. XU RH, Wong ELY. Involvement in shared decision-making for patients in public specialist outpatient clinics in Hong Kong. Patient Prefer Adherence. 2017;11:505-512. doi:10.2147/PPA. $\mathrm{S} 134792$

22. Hong Kong Special Administraion Region (HKSAR) Government Census and Statistics Department \&Hong Kong Census and Statistics Department. Hong Kong Population Projections 20152064. Hong Kong Census and Statistics Department; 2015.
23. Orr JG, Homer T, Ternent L, et al. Health related quality of life in people with advanced chronic liver disease. $J$ Hepatol. 2014;61:1158-1165. doi:10.1016/j.jhep.2014.06.034

24. Simmons LA, Wolever RQ, Bechard EM, Snyderman R. Patient engagement as a risk factor in personalized health care: a systematic review of the literature on chronic disease. Genome Med. 2014;6:113. doi: $10.1186 / \mathrm{gm} 533$

25. Xu RH, Cheung AW, Wong EL. Development and validation of an instrument to measure patient engagement in Hong Kong Special Administrative Region, China. Patient Prefer Adherence. 2018;12:1667-1675. doi:10.2147/PPA.S176067

26. Rabin R, Oemar M, Oppe M, Janssen B, Herdman M. EuroQol, EQ5D-5L User Guide. Avaliable from: https://euroqol.org/docs/EQ-5D5L-User-Guide.pdf. Accessed August 19, 2019.

27. Tran BX, Ohinmaa A, Nguyen LT. Quality of life profile and psychometric properties of the EQ-5D-5L in HIV/AIDS patients. Health Qual Life Outcomes. 2012;10:1. doi:10.1186/1477-7525-10-132

28. Wong ELY, Ramos-Goñi JM, Cheung AWL, Wong AYK, RiveroArias O. Assessing the use of a feedback module to model EQ-5D-5L health states values in Hong Kong. Patient. 2018;11:235-247. doi:10.1007/s40271-017-0278-0

29. Venables WN. Modern Applied Statistics with S. New York: Springer; 2002.

30. Rademakers J, Nijman J, Van DerHoek L, Heijmans M, Rijken M. Measuring patient activation in the Netherlands: translation and validation of the American short form Patient Activation Measure (PAM13). BMC Public Health. 2012;12:1. doi:10.1186/1471-2458-12-577

31. Schneider E, Squires D. From last to first - could the U.S. health care system become the best in the world? N Engl J Med. 2017;337:901904. doi:10.1056/NEJMp1708704

32. Coulter A. Engaging Patients in Health Care. Picker Institute Europe. 2006. Available from: https://www.picker.org/wp-content/ uploads/2014/10/Engaging-patients-in-their-healthcare-how-is-theUK-doing.pdf. Accessed August 19, 2019.

33. Coulter A. Engaging Patients in Healthcare. Maidenhead: Open University Press; 2011.

34. James J. Health Policy Brief: Patient Engagement. Health Affairs; 2013. Available from: https://www.healthaffairs.org/do/10. 1377/hblog20130215.028299/full/. Accessed August 19, 2019.

35. Cheung FM, Cheung FM, Halpern DF. Women at the top: powerful leaders define success as work + family in a culture of gender. $\mathrm{Am}$ Psychol. 2010;65:182-193. doi:10.1037/a0017309

36. Goldman D, Smith JP. The increasing value of education to health. Soc Sci Med. 2011;72:1728-1737. doi:10.1016/j.socscimed.2010.11.008

37. Field MG. The health system and the social system. In: Sokołowska M, Hołówka J, Ostrowska A, editors. Health, Medicine, Society. Netherlands: Springer; 1976:315-329. doi:10.1007/978-94-010-1430-4_20

38. Boutin M, Dewulf L, Hoos A, et al. Culture and process change as a priority for patient engagement in medicines development. Ther Innov Regul Sci. 2017;51:29-38.

39. Holzmueller C, Wu A, Pronovost P. A framework for encouraging patient engagement in medical decision making. J Pateint Saf. 2012;8:161-164. doi:10.1097/PTS.0b013e318267c56e

40. Kaplan E, Kahan M, Lauren H, Suchday S. Does social comparison affect the socioeconomic status-health relationship? Psychol Heal. 2011;26:258-259.

41. Begley C, Basu R, Lairson D, et al. Socioeconomic status, health care use, and outcomes: persistence of disparities over time. Epilepsia. 2011;52:957. doi:10.1111/epi.2011.52.issue-5

42. Hoi LV, Chuc NTK, Lindholm L. Health-related quality of life, and its determinants, among older people in rural Vietnam. BMC Public Health. 2010;10:549. doi:10.1186/1471-2458-10-549

43. Elbert NJ, van Os-Medendorp H, van Renselaar W, et al. Effectiveness and cost-effectiveness of ehealth interventions in somatic diseases: a systematic review of systematic reviews and meta-analyses. $J$ Med Internet Res. 2014;16:e110-e110. doi:10.2196/jmir.2790 
44. Hibbard BJH, Greene J. What the evidence shows about patient activation: better health outcomes and care experiences; Fewer data on costs. Health Aff. 2013;32:207-214. doi:10.1377/hlthaff.2012.1061

45. Shortell S, Poon BY, Ramsay PP, et al. A multilevel analysis of patient engagement and patient-reported outcomes in Primary Care Practices of Accountable Care Organizations. J Gen Intern Med. 2017;32:640-647. doi:10.1007/s11606-016-3934-5
46. Aung E, Donald M, Williams GM, Coll JR, Doi SAR. Influence of patient-assessed quality of chronic illness care and patient activation on health-related quality of life. Int J Qual Heal Care. 2016;28:306310. doi:10.1093/intqhe/mzw023

\section{Publish your work in this journal}

Patient Preference and Adherence is an international, peer-reviewed, open access journal that focusing on the growing importance of patient preference and adherence throughout the therapeutic continuum. Patient satisfaction, acceptability, quality of life, compliance, persistence and their role in developing new therapeutic modalities and compounds to optimize clinical outcomes for existing disease states are major areas of interest for the journal. This journal has been accepted for indexing on PubMed Central. The manuscript management system is completely online and includes a very quick and fair peer-review system, which is all easy to use. Visit http:// www.dovepress.com/testimonials.php to read real quotes from published authors. 